Aus dem pharmakognostischen Institute der Universitat Wien.

\title{
Der Hydrastin- und Berberingehalt in Oesterreich (Korneuburg) kultivierter Hydrastis canadensis und uber quantitative Berberinbestimmung.
}

Von Richard Wasicky u. Marianne Joachimowitz.

(Eingegangen den 6. X. 1917.)

Die Kulturversuche, die E. Se $n f^{(i)}$ mit der Hydrastis canadensis in Korneuburg bei Wien angesiellt hat, legen in eindeutiger Weise die Bedingungen klar, unter denen die Stammpflanze der so wertvollen Droge in Mitteleuropa anzubauen und ein gehalivolles, das aus Amerika eingeführ'e Rhizom an Güte übertreffendes Produke zu erzielen ist. Als Alkaloidgehalt führt S e $\mathbf{f} f \dot{r}$ in der bezüglichen Arbeic für die Rhizome 3,77\% Hydrastin, $3 \%$ Berberin, für die Nebenwurzeln 1,9\% Hydrastin, $2 \%$ Berberin an. Bei der diesen Daten zugrunde liegenden chemischen Untersuchung der Droge, die im pharmakognostischen Universitätsinstiuuce in Wien vorgenommen wurde, ergaben sich einige beachtenswerte Tatsachen, über die im nachfolgenden berichtet werden soll.

Das aus den Korneuburger Kuliuren stammende Pflanzenmaterial besiand aus den lebenden unierirdischen Organen der Gelbwurz. Nach sorgfäliiger Reinigung wurden die zahlreichen Wurzelfasern knapp am Wurzelstock abgeschnitien und Wurzelstöcke und Wurzelfasern getrenni getrocknet und der Analyse zugeführt. Der Hauptieil der Droge wurde der Lufitrocknung unterworfen, ein kleinerer Teil bei $100^{\circ}$ im Trockenschrank getrocknet, um den Einfluß einer künstlichen Trocknung bei erhöhter Temperatur kennen zu lernen. Ohne genaue zahlenmäßige Wiederga be der erhaltenen Ergebnisse sei bemerki, daß weder im Hydrastinnoch im Berberingehalt der beiden Drogenanteile wesentliche Differenzen festgestelli werden konnten, so daB wir mit allem Vor. behalt für eine künstliche Trocknung einireten, falls die eingehendere von uns fortgeseizte Prüfung keine nachteiligen, durch die künstliche Tracknung bedingten Aenderungen im Eigenschaftenkomplex der Droge feststellen sollte.

1) E. S enft, Pharm. Post L. (1917), S. 2.

Arob. d. Pharm. OOLV. Bdo. 8 Hott. 
Die Methodik der Hydrastinbestimmung bedarf keiner wt $i$. teren Ausführung, da sie in den letzten Jahren durch die Arbeiten verschiedener Forscher ${ }^{1}$ ) eine genaue, kritische Bearbeitung erfahren hat und die allgemein geübten Verfahren die an sie gestellten Ansprüche vollauf befriedigen. Wir bedienten uns für die Bestimmung der Methode Keller-Rusting - From me $\mathrm{e}^{2}$ ) und der von $\mathrm{v}$ a $\mathrm{n}$ der $\mathrm{W}_{\mathrm{a}} \mathrm{a}^{3}$ ) eingehaltenen Arbeitsweice. Die gewonnenen Alkaloidmengen erreichten bei beiden. Verfahren fast die gleiche Höhe und betrugen, wie schon erwähnt, bei den luftgetrockneten Wurzelstöcken $3,77 \%$, bei den Nebenwurzeln $1,9 \%$ Hydrastin.

Nicht so glatt verlief die Bestimmung des Berberins, da für sie erst eine Methode geschaffen werden mußte. Die in der Literatur bekannten Bestimmungen besitzen ihren Geltungsbereich für jene Fälle, in denen das Berberin fast das einzige Alkaloid in der Droge bildet (Berberis) oder, wenn sie schon auf die Hydrastis Anwendung gefunden haben, mußte man eine gewisse Ungenauigkeit in Kauf nehmen. Die Methode nach $S \mathrm{ch}$ w i cker a $t h-L$ in d e (Fällung des Berberins als Sulfat) und jene nach Gordin und Prescot (Fällung als Acetonberberin) liefern ungenaue Ergebnisse, da bei beiden Verbindungen die Fällung keine vollständige ist4).

J. Troeger und $\mathrm{O}$. Lind $\mathrm{e}^{5}$ ). haben vorgeschlagen, $\beta$-naphthalinthiosulfonsaures Kalium für die Berberinbestimmung heranzuziehen unter Verwertung des Umstandes, daß eine Lösung des genannten Reagens im Ueberschu $B$ zu einer Lösung von Berberinhydrochlorid hinzugefügt, letzteres quantitativ ausfällt. Die beiden Verfasser haben eine im ungefähren Verhältnis $1: 300$ hergestellte Lösung des Salzes unter Zuعatz von etwas Stärkelösung titrimetrisch mit $1 / 100-\mathrm{N}$.-Jodlösung ausgewertet. Sodann wurde das in der zu prüfenden Lösung entstandene naphthalinthiosulfonsaure Berberin abfiltriert und das klare und farblose Filtrat, welches den Ueberschuß des Reagenses enthielt; auf Eintritt der Blaufärbung mit Jodlösung titriert. Aus den erhaltenen Zahlen be-

1) Es sei hier verwiesen auf: J äg g i, Schw. Wchgchr. f. Chem. u. Pharm. XLVIII., S. 629; H. D i ch g a n s, Apoth.-Ztg. 29: (1914), S. 498; L. D \&́ vid, Pharm. Post XLVIII. (1915), S. 1; J. va n d er Wa a l, Pharm. Weekbl. 52. (1915), S. 1423. In den angeführten Arbeiten ist auch die ältere Literatur zitiert.

*) Sieho Jahresber. von Caesar \& Loretz in Halle a. $\mathbf{S}$.

) 1.0 .

4) Siehe J. Katz, Pharm. Zentralh. XXII. (1901), S. 283.

9) J. T r o e g er und O. L i n d e, dieses Archiv 288. (1900), \$. 4 
rechneten die Verfasser den Berberingehali und crzielien sehr zufriedenstellende Ergebnisse. Die von uns durchgeführten Versuche mit $\beta$-naphthalinthiosulfonsaurem Kali, das wir dem liebenswürdigen Entgegenkommen des Herrn Geh. Medizinalrates Prof Dr. H. B e c u r t s verdanken, ergaben auch uns bei reinen Berberinsalzlösungen aukgezeichnete Resultate. Für die Bestimmung des Berberins in der Hydrastiswurzel erwies sich das elegante Verfahren leider nicht anwendbar, da es für die Titration mit Jod, wie wir uns durch Versuche tiberzeugten, eine genau neuirale Alkaloidsalzlösung erfordert, der Erlangung einer solchen aber bei der analytischen Bestimmung sich unüberbrückbare Hindernisse in den Weg stellen.

Dagegen eignet sich $\beta$-naphthalinthiosulfoncaures Kali besonders gut für den mikrochemischen Nachweis einiger Alkaloide, worüber wir an anderer Sielle berichten werden. Setzt man eine wässerige Lösung des Reagens einem Trockenschnitt des Hydrastisrhizoms selbst $z u$, erwärmt und läßt erkalten, so scheidet sich die Hydrastinverbindung in ganz kleinen Körnchen, das Berberin in schönen, aus gelben, spitzen Nadeln sich zusammensetzenden Krystallaggregaten aus. Während für die Hydrastis der geschilderte Nachweis der Alkaloide keine Vorteile gegenüber den schon bekannten Methoden bietet, bedeutet das Verfahren für andere Drogen eine wertvolle Bereicherung der Kenntnisse, die wir über ihr mikrochemisches Verhalten besitzen.

Von den für die Bestimmung des Berberins in der Hydrastis angegebenen Methoden sind zweifellos die beiden von $\mathrm{H}$. M. G or $\mathrm{d} \mathrm{in}^{\mathbf{1}}$ ) in Band 239 dieser Zeitschrift veröffenilichten als die brauchbarsten zu bezeichnen. Die eine der beiden, die der Verfasser angewendet wissen will, wenn es auf große Genauigkeit nicht ankommt, fällt das Berberin im alkoholischen Hydrast isextrakt als عaures Sulfat. Letzteres wird in wässeriger Lösung mit Jodkali umgesetzt, der entstandene Berberinhydrojodidniederschlag abfiltriert und im Filtrat die in Freiheit gesetzte Schwefeleäure tịtrimetrisch bestimmt. Beim zweiten Verfahren werden $20 \mathrm{~g}$ der Droge mit heißem Alkohol extrahiert, das Extrakt auf etwa $20 \mathrm{ccm}$ eingeengt und mit Wasser auf $500 \mathrm{ccm}$ verdünnt. Die Flüssigkcit echüttelt man mit $2-3 \mathrm{~g}$ Talkum 15-20 Minuten lang und filtriert. $250 \mathrm{ccm}$ des Filtrates werden mit 15-20 ccm 20\%iger Jodkaliumlösung versetzt und der entstandene Niederschlag in ein Kölbchen mit Wasser gespült. Die Flussigkeit schüttelt man nún mit dem halben Volumen Aceton

1) H. M. G ordin, dieses Archiv 239. (1901), S. 638. 
durch 10 Minucen, setzt $5 \mathrm{ccm} 10 \%$ ige Natronlauge zu und schüttelt so lange, bis das gelbe Hydrojodid verschwunden ist, erwärmt allenfalls auf $50-60^{\circ}$, bis alles Hydrojodid in Acetonberberin verwandelt ist. Nach dem Erkalten setzt man soviel Wasser zu, daß der Acetongehalt etwa $1 / 9$ der Flüssigkeit ausmacht und stellt über Nacht beiseite. Der Niederschlag wird in einem G o o c h'schen Platintiegel gesammelt, mit Wasser gut gewaschen, über Nacht im luftleeren Raum gehalten, dann bei $105^{\circ}$ getrocknet und gewogen Dem erhaltenen Resultat zählt man für jeden Kubikzentimeter Mutterlauge $0,0000273 \mathrm{~g}$ Berberin zu. Liefert nun auch die langwierige und sehr mühevolle Methode, bei der man den Gang peinlichst einhalten muß, genauere Resultate als die bisher erwähnten, so sieht doch die angewandte Mühe in gar keinem Verhältnis zum erzielten Ergebnis; denn auch hier wird die Genauigkeit durch einige Umstände beeinirächtigt. Da das Berberinhydrojodid in Alkohol nicht unlöslich ist, muß der Alkohol möglichst entfernt werden. Da aber auch im eingeengten Extrakt noch ein wenig Alkohol zurückbleibt, so ist die Fällung des Berberinhydrojodids keine quantitative. Ein zweiter Fehler entsteht dadurch, da B beim Abfiltirieren der mit Talkum geschüttelten Flüssigkeit Berberin im Niedersehlag zurückgehalten wird und durch Waschen mit Wasser allein nicht in Lösung zu bringen ist, sondern erst auf Alkoholzusatz. Eine weitere Fehlerquelle bedeutet bei der Empfindlichkeit des Alkaloides gegenüber Erwärmen mit Lauge das Arbeiten in alkalischer Lösung unter Anwendung erhöhter Temperaturen. Schließlich ist noch darauf hinzuweisen, daß die Korrektur durch Hinzuzählen einer bestimmten Menge Berberins je nach dem angewandten Volumen Mutterlauge, die durch die Löslichkeit des Acetonberberins in Acetonwasser bedingten Fehler naturgemäß nicht einwandfrei ausschaltet.

In der Wurzelrinde von Berberis vulgaris bestimmt E. R i c h te $\mathbf{r}^{1}$ ) den Berberingehalt durch Fällung der ätherischen Lösung des Berberinals mit einer ätherischen Pikrolonsäurelösung. Auf die Methode, die sich für die Bestimmung des Berberins erst nach der Trennung von den übrigen Alkaloiden eignet, wird später eingegangen werden.

Fehlerhaft und ganz unbrauchbar ist die Methode, die L. Dávid $\left.{ }^{2}\right)$ für die Berberinbestimmung im Hydrastisextrakt angegeben hat. Nach ihm werden $2 \mathrm{~g}$ Extraki in einem Schtitteltrichter

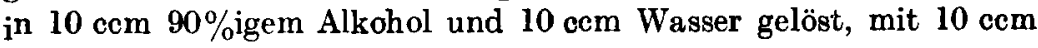

1) E. R i c h t e r, dieses Archiv 252. (1914), S. 192.

2) L. D \& vi d, Pharm. Post XLVIII. (1815), S. 1 . 
Kaliumwismutjodidlösung versetzc und nach Zugabe von $60 \mathrm{cem}$ Essigäther eine Minute lang stark geschüttelt. Nach halbstündigem Stehen wird abfiltriert, zweimal mit $5 \mathrm{ccm}$ Essigäther der Schü:teltrichter, dann noch mit $5 \mathrm{ccm}^{\circ}$ Essigäther das Filter gewaschen. Filter und Niederschlag werden in einem weithalsigen Gefäß mi Natronlauge anter Zueatz von Kochsalz sĩark geschüûtelt, dann das Berberinal mic Aether-Chloroform $(50+50) 15$ Minuten geschüttelt und $80 \mathrm{~g}$ Aether-Chloroformlösung abfiliriert. Schließlich wird die Alkaloidlösung im Vakuum abgedampft und der Rückstand bei $90^{\circ}$ bis zur Gewichîskonsianz getrocknet und gewogen. Ohne in eine die Einzelheiten berücksichtigende Kritik einzugehen, sei hervorgehoben, daß der Berberinniederschlag mi wismutjodid in Essigäther-Alkohol-Wasser-Mischung merklich löslich ist und sich daher ein Teil des Berberins der Bestimmung entzieht. Nebenbei sei ferner darauf hingewiesen, daß der schließliche Abdampfrückstand nicht aus Berberinal ${ }^{1}$ ) besteht, wie $D$ á $v$ id meint. sondern aus Chloroform-Berberin, und auch aus diesem Grunde die von ihm angestellte Berechnung einer Korrektur bedarf Daß unier solchen Umständen der Verfasser mit seiner eigenen Meíhode bei der Bestimmung des reinen kalzeauren. Berberins Verlusîe von 14,3-21;67\% erhält, ist erklärlich; ébenso daß bei der Extrakibestimmung sich Unterschiede bis zu $54-55 \%$ ergeben. Wie der Verfasser dann noch im Schlußeatze davon sprechen kann; daß es ihm ". . gelang, ein Verfahren zur Berberin-Bestimmung im Extractum hydrastis auszuarbeiten", erscheint nicht verständlich.

Die bisherigen Ausführungen lassen erkennen, daß die $\mathrm{Fr}$ gebnisse der Berberinbestimmung nach den geschilderten Meihoden keineswegs befriedigen. Dagegen gelang es uns, das gewünschte Ziel mittels einer Methode zu erreichen, auf deren Eignung für die Trennung von Strychnin und Brucin der eine von uns ${ }^{2}$ ) schon früher einmal hingewiesen hatte. Im Wesen beruht die Methode auf dor verschiedenen Löslichkeit der Alkaloidfällungen mit dem M a y $\urcorner \mathrm{r}$ sehen Reagens in Alkohol. Während der mit Kalium-Quecksilberjodidlösung erhaltene Hydrastinniederschlag schon von der gleichen Menge 95\%igem Alkohol spielend gelösì wird, bleibt die Lösung der Berberinfällung mit dem gleichen Reagens auch bei Zusaîz des 20 fachen Alkoholvolumens im Verhältnis zur angewandien Flüssigkeit aus. Seite 54.

1) In der zitierten Arbeit durchwegs mit Berberal bezeichnet.

$\left.{ }^{2}\right)$ R. Wa s i e k y, Ztschr. d. Allg. österr. Ap.-Ver. LTT. (1914), 
In seinen Einzelheiten gestaltete sich der Gang für die Bestimmung eines Berberinsalzes folgendermaßen: Eine gewogene Menge salzkauren Berberins $(0,2 \mathrm{~g})$, das wir durch Umkrystallicieren reinigten und von dessen Reinheit wir uns durch Bestimmung des Wasser- und Chlorgehaltes überzeugten, wurde in konzentriertem Alkohol gelöst und mit angesäuertem $M$ a y e r'schen Reagens im Ueberschuß (bis auf weiteren Zusatz keine Fällung erfolgte) versetzi. Der entstandene Niederschlag wurde abfiltriert und samt dem Filter mit $20 \%$ iger Kalilauge (etwa $20 \mathrm{ccm}$ ) in einen Schütteltrichter gebracht. Nach 5 Minuten Schütteln wurden $150 \mathrm{ccm}$ Aether und $5 \mathrm{~g}$ Natriumchlorid. hinzugefügt, 1/2 Stunde geschüttelt, durch 5 Stunden stehen gelassen, schließlich nochmals durch 5 Minuten geschüttelt. Hier wäre zu bemerken, daß wir zur Zersetzung des Niederschlages auch alkalische Zinnoxydullösung, ferner Natronlauge versuchien, freilich mit negativem Erfolg, da in der Kälte die Zersetzung der Berberinfällung auch bei anhaltendem und energischem Schütteln keine vollständige war und in der Wärme noch niedrigere Werte für das Alkaloid erhalten wurden. Zweifellos erfolgt in der Wärme unter dem EinfluB der Lauge eine Zersetzung des Berberins, auf die $\mathrm{Gad}$ a $\mathrm{m} \mathrm{e}^{1}$ ) hingewiesen hat, und die nach seinen Untersuchungen in der Richtung des Dihydroberberins und Oxyberberins verläuft.

Der Versuch, aus der ätherischen Berberinallösung das Berberin einfach durch Abdestillieren des Aethers und nach dem Trocknen durch Wägung zu bestimmen, ergab ganz unbrauchbare Resultate, da die erhaltenen Analysenwerte zu stark voneinander abwichen. Wir wählten demnach für den leízten Abschnitt der Berberinbestimmung das schon oben erwähnte, von E. R i ch t er für die Berberinbesíimmung in der Wurzelrinde der Berberis vulgaris angewandte Verfahren. R i c h t e r stellt eine alkoholische Extraktlösung der Droge her, befreit von Alkohol, verseizt die wässerige Alkaloidlösung mit Natronlauge und schüctelt mit Aether aus. Die ätherische Berberinlösung wird mit einer ätherischen Pikrolonsäurelösung versetzt, der entstehende Niederschlag auf einem G o o ch-Tiegel abgesaugt, mit einer Mischung von Aether und Alkohol nachgewaschen, getrockne i und gewogen. Bei unserer Alkaloidbestimmung pipettierten wir dem entsprechend $100 \mathrm{ccm}$ der ärherischen Berberinallösung aus dem Schütteltrichter ab und versetzten mit einer gesättigten ätherischen Pikrolonعäurelösung, bis sich die Flüssigkeit über dem zusammengeballten Berberin-

1) J. G a d a m e r, dieses Archiv 248. (1905). S. 31. 
pikrolonat nicht mehr trübíe. Der Niederschlag wurde auf einem G o o c h-Tiegel abgesaugt, mit Aether gewaschen, bei $110^{\circ}$ getrocknet und gewogen. Aus dem Berberinpikrolonat (Molekulargewicht 600,25) wurde das Berberin berechnet. Die bei einer Anzahl von Bestimmungen so erhaltenen Zahlen ergaben übereinsíimmende, sehr zufriedenstellende Resultate. Auch aus Mischungen von Hydrastinhydrochlorid und Berberinhydrochlorid wurde auf diese Weise das Berberin vollständig wiedergewonnen, so daß wir uns nunmehr der Droge selbst zuwenden konnten.

Wir extrahier'en die gepulverte Droge im S o x h l e $\hat{t}$ - Apparat mit 95\%igem Alkohol, bis die Flüssigkeit ganz farblos ablief, Dann wurde die Lösung durch Abdampfen auf dem Wasserbade soweit eingeengt, da $5 \mathrm{~g}$ Droge ungefähr $50 \mathrm{~g}$ Alkohol entsprachen -, und darauf mit angezäuertem Ma y er'schen Rcagens im Ueberschuß versetzt. Der abfiltrierte Niederschlag wurde mit Alkohol, dem ein wenig Kalium-Quecksilberjodidlösung zugesetzt war, gewaschen, bis sich im Filtrat kein Alkaloid mehr nachweisen ließ, was nach dem dritten Aufgießen aufs Filter bereits der Fall war. Sodann wurde mit Wasser und wenig $M$ a y e $r$ 'schem Reagens gewaschen, schließlich samı Filter mit $20 \mathrm{ccm}$ Kalilauge in einen Schüttel. trichter gebracht und weiter wie oben behandeli. Um die langwierige Soxhlet-Extraktion zu umgehen, wurden auch noch andere Extraktionsverfahren angewandt. Prakĭisch bewährie es sich am besten, die gepulverte Droge mit der 10 fachen Menge konzentrierten Alkoho's anzuseizzen und unter zeitweiligem Umschưtteln durch 48 Stunden stehen zu lassen. Von der klaren, über dem Boden stehenden Extraktionsflussigkeit wurde ein aliquoter Teil abgemessen und der gleichen Behandlung wie das S oxh le t - Extrakt unterzogen. Die so nach den beiden Methoden gewonnenen Resultate unterschieden sich voneinander nicht, und zwar fanden sich bei der Untersuchung der in Oesterreich kultivierten Hydrasíis die eingangs erwähnten Zahlen für Berberin.

Noch ein anderer Weg ist gangbar zur Erlangung genauer Werte bei der Berberinbestimmung in der Hydrastisdroge. Die von dem amerikanischen Arzneibuche zur Hydrastinreinigung verwertete Eigenschaft des Berberins, in wässe:igen Lösungen durch überschüssiges Jodkalium quantitativ ausgefällı zu werden, ermöglicht es, die beiden Hauptalkaloide der Hydrastis zu trennen. Wie früher ausgeführt wurde, bedienten sich auch Gordin und Prescoit bei ihrem Verfahren des Jodkaliums zu dem gleichen Zwecke, ohne freilich dem Umstande, daß kein Alkohol vorhanden sein und daB die Ueberflahrung des Berberinhydrojodids in Aceton- 
berberin und die Isolierung des letżteren nicht den Anspruch einer: genauen quantitativen Bestimmung erheben darf, gebührende Beachtung zu schenken. Bei der von uns gewählten Mechodik wurden $6 \mathrm{~g}$ gepulverter Droge mit $60 \mathrm{~g}$ Wasser durch $1 / 1$ Stunde auf dem Wasserbade erhitzt. Das verdampfte Wasser wurde ergänzi und die Probe durch 48 Stunden unter zeitweiligem Schütteln stehen gelassen. Von der sodann abgepreßien Flüssigkeit wurden $50 \mathrm{~g}$ abfiltriert und mit überschüssiger Jodkaliumlösung versetzt. Der abfiltrierte Niederschlag wurde mit verdünnter Jodkaliumlösung gewaschen, darauf Filter und Nicderschlag zusammen mit $20 \mathrm{ccm}$ $20 \%$ iger Kalilauge und soviel festem Kochsalz, daß ein Teil ungelöst blieb, und $150 \mathrm{ccm}$ einer im Verhältnis $10: 1$ hergestellten AetherAlkoholmischung in einen Schütteltrichter gebracht'. Nach $1 / 4$ stündigem energischen Schütteln und Absetzenlassen wurden $100 \mathrm{ccm}$ der ätherischen Berberinallösung mit ätherischer Pikrolonsäurelösung, dann weiter wie beim Verfahren mit Kalium-Quecksilberjodid behandelt. Die bei Vergleichsbestimmungen mit Jodkalium erhaltenen Werte wiesen mit den nach dem crsten Verfahren erzielten eine hinlängliche Uebereinstimmung auf, so daß auch die Methode in der vorliegenden Form den gewünschten Anforderungen gerecht wird.

Theoretisches, vielleicht aber auch praktisches Interesse bot es, die Blätter der Gelbwurz, die uns reichlich zur Verfügung standen, auf ihren Alkaloidgehalt, vor allem aber auf ihren Hydrastin- und. Berberingehalt zu untersuchen. Unseres Wissens stehen in der genannten Richtung sich bewegende Analysen vollständig aus

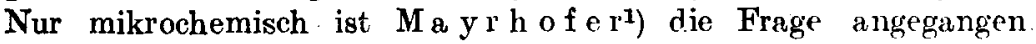
Mittels Pikrolonsäure gelang es ihm den Nachweis zu führen, dals im Blatt und. Stengel der Gelbwurz Hydrastin und Berberin vorhanden sind, daß der Stengel weniger Alkaloide enihält als die unterirdischen Organe, die Blätter wieder weniger als die Stengel. Aus den Korneuburger Kulturen erhielten wir dic Sproßachsen mit Blättern im getrockneten Zustande. Es wurden die Blattspreiten von den Stielen abgetrennt und es kamen Spreicen und Sproßachsen mit den Blattstielen gesondert zur Untersuchung. Es zeigte sich, daß sich für sie die gleiche Technik anwenden ließ wie für die Wurzelstöeke und Wurzeln. Nur wurden entsprechend größere Mengen verarbeitet, um auch die Identität der Alkaloide festzustellen. Die Blattspreiten enthielten 0,77\% Hydrastin und 0,55\% Berberin, die Blattstiele und. Sproßachsen 1,12\% Hydrasiin

1) A. Ma yr h of or, Pharm. Post 47. (1014), S. 547. 
R. Wasicky u. M. Joach imowitz: Hydrastis canadensis. 505

und 1,18\% Berberin. Die gefundenen Analysenwerîe bestätigen demnach den mikrochemischen Befund. $M$ a y r h of er s. Sie lassen ferner ersehen, daß auch die oberirdischen Organe der Gelbwurz erhebliche Mengen Alkaloide führen, wenn auch in geringerem Ausmaße als die unterirdischen. Bläıter und Sproßachsen stellen sich demnach als ziemlich wertvolles Material dar, und es wird sich empfehlen, sie gleichfalls therapeutisch zu verwenden, falls, wie $z u$ erwarten steht, die Kultur sich bei uns einbürgert.

\section{Schlußbemerkungen und Zusammenfassung.}

Für eine genaue Berberinbestimmung reichen die bis jetzt bekannten Methoden nicht aus, wenn man auch für die Zwecké der Praxis gut das Aus!angen mit der einfachen Methode von S c h wi ckerath und Linde (Fällung des Berberins als Sulfat) findet. Genauere Resuliate würde, und zwar mit demselben Aufwand an Zeit und Mïhe, eine Gesamtalkaloidbestimmung liefern, die sich an die ohnehin not wendige Hydrastinbestimmung anschlie Ben und die mittels Pikrolonsäure durchgeführt würde, etwa in der Weise, wie dies H. Matthes und $O$. Ramsted $t^{1}$ ) für das Hydrastisextrakt versucht haben. Die Differenz aus der Gesamtmenge der Alkaloide und dem Hydrasín würde auf das Berberin entfallen, wobei das quaniitativ unbedeutende Canadin und. Mekonin vernachlässigt werden.

Eine genaue Berberinbestimmung in der Gelbwurz wird durch die Verwertung der Tatsache ermöglicht, daß der mit KaliumQuecksilberjodidlösung erhaltene Berberinniederschlag in Alkohol unlöslich ist, dagegen der Hydrastinniederschlag sich in Alkohol sehr leicht löst. Unter Berücksich igung der durchgeführten Versuche wird folgendes Bestimmungsverfahren für Berberin vor-

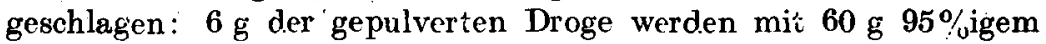
Alkohol durch 48 Siunden unter zeitweiligem Aufschütteln stehen gelassen, sodann $50 \mathrm{~g}$ des Extrakies abfiltriert und mit angecäuertem $\mathrm{M}$ a y e r'schen Reagens im Ueberchuß versetzt (50 $\mathrm{g}$ ist mehr als ausreichend). Der entstandene Niederschlag wird abfiltrier $t$, durch dreimaliges Aufgießen von 50\% igem Alkohol, der ein wenig Ma yer'sches Reagens enthält, dann mit kalium-quecksilberjodidhaltigem Wasser gewaschen und samt Filter in cinen Schütteltrichter gebracht und 5 Minuten geschüttelt. Nach Zusatz: von $5 \mathrm{~g}$ Kochsalz und $150 \mathrm{ccm}$ Aether wird durch eine 1/2 Stunde

1) H. Matthos und O. Ramatedt, dieses Archiv 845. (1907), Seite 112. 
geschüttelt, 5 Síunden stehen gelassen, schließlich nochmals durch 6 Minuten geschüttelt. $100 \mathrm{ccm}$ der klaren ätherischen Berberinallösung werden abpipettiert und mit überschüssigrr ätherischer Pikrolonsäurelösung versetzt. Das entsiandene Berberinpikrolonat saugt man auf einem Gooch - Tirgel ab, wäscht mit Aether nach, trocknet bei $110^{\circ}$ und wägt. Aus den Molekulargewichten für Berberinpikrolonat 600,25 , Berberin 353,26 und aus dem ermittelten Gewicht berechnet man das Berberin.

Zur Trennung des Berberins und Hydrastins läßt sich an Stelle des Kaliumquecksilberjodids auch Jodkalium in wässeriger Lösung verwenden. $6 \mathrm{~g}$ Droge werden mit $60 \mathrm{~g}$ Wasser durch $1 / 4$ Stunde auf dem Wasserbade erhitizt und nach Ercaíz des verdampfien Wassers durch 48 Stunden stehen gelassen. $50 \mathrm{~g}$ des abgepreßten und abfiltrierien Extraktes vercetzzt man mî́ überschüssiger Jodkaliumlösung, filtriert das gefällte Berberinhydrojodid ab, wäscht mit jodkalihaliigem Wasser nach, solange das Filtrat noch Alkaloidreakíionen zeig $t$, und behandel $t$ dann den Niederschlag genau sowie den mit Kaliumqueck:ilberjodid erhalienen, nur daß man sich zum Ausschütteln des Berberinaldehydes einer 10:1 hergestellten Aether-Alkoholmischung bedient.

Unter Anwendung der geschilderten Me. thoden fur die Berberinbestimmung und der Methoden von Keller-Rusting-Fromme und van der Waal für die Hydrastinbestimmung wurde in Korneuburg bei Wien kultivierte Hydrastis canadensis untersucht. Es ergaben sich folgende Alkaloidgehalte: für die Neben. wurzeln $1,9 \%$ Hydrastin, $2 \%$ Berberin, für die Wurzelstöcke $3,77 \%$ Hydrastin, $3 \%$ Berberin, für die Blattspreiten $0,77 \%$ Hydrastin, $0,55 \%$ Berberin, für die Sproßachsen und Blattstiele $1,12 \%$ Hydrastin und $1,18 \%$ Berberin. Die in den oberirdischen Organen der Hydrastis vorhandenen erheblichen Alkaloidmengen lassen es ratsam erscheinen, auch die bei der Kultur des. Hydrastisrhizoms geernteten Blätter und Sproßachsenalswertvolle Droge in therapeutis: 